\title{
Estrategias para la enseñanza del autoexamen del seno a mujeres en edad reproductiva*
}

\author{
Doris 0 rtega-Altamirano, M. en C., (1) Lizbeth López-Carrillo, Ph. D., ${ }^{(2)}$
}

Malaquías López-Cervantes, Ph. D. . $^{(1)}$

\section{Ortega-Altamirano D, López-Carrillo L, López-Cervantes $M$. \\ Estrategias para la enseñanza \\ del autoexamen del seno a mujeres en edad reproductiva. \\ Salud Publica Mex 2000;42:17-25.}

\section{Resumen}

Objetivo. Disponer de una estrategia efectiva de enseñanza de conocimientos en cáncer de mama (CM) y en la técnica del autoexamen del seno (AES) para las mujeres mexicanas de entre 12 a 47 años de edad. Material y mé todos Entre abril de 1996 y julio de 1998, en la ciudad de Cuernavaca, Morelos, México, se diseñaron y evaluaron dos estrategias de enseñanza del AES y sus materiales didácticos con base en las propuestas de las mujeres participantes en el inicio de este estudio. A mbas estrategias consistieron en la presentación de un folleto y una cinta de video; en la primera, una enfermera en salud pública condujo la sesión; en la segunda, una sobreviviente de C M. Además, en la segunda estrategia se adicionaron unas diapositivas y unos modelos de senos de silicón. Ambas estrategias se compararon con una tercera que se extrajo de las normas de la Secretaría de Salud, consistente en la presentación de una hoja impresa y una cinta de video por parte de una enfermera. Participaron 149 mujeres, asignadas aleatoriamente a cada estrategia. La efectividad de éstas se evaluó por los cambios en los conocimientos en AES y CM, y en la destreza para identificar mayor número de abultamientos en un modelo de seno de silicón tamaño natural, medidos antes y después de la capacitación. Se realizaron para el análisis estadistico pruebas $t$ de Student, análisis de varianza, $\chi^{2}$ de MCN emar, la de Pearson y regresión lineal múltiple. Resultados Con las tres estrategias se elevaron aproximadamente $30 \%$ los conocimientos de las participantes sobre

\author{
Ortega-Altamirano D, López-Carrillo L, \\ López-Cervantes M. \\ Strategies for breast \\ self-examination training to women \\ of reproductive age. \\ Salud Publica Mex 2000;42:17-25.
}

\begin{abstract}
A bstract
Objective.To develop an effective strategy to inform Mexican women between 12 and 47 years of age about breast cancer (BC) and train them to perform breast self-examination (BSE). Material and methods Between A pril, 1996 and July, 1998, two different strategies to teach BSE were designed and evaluated in Cuernavaca, State of Morelos, Mexico. These strategies included teaching materials and were based on opinions of women participating in the first stage of this study. Both strategies consisted of the presentation of a leaflet and a video. The first strategy was delivered by a public health nurse in charge of leading the session. The second one was presented by a BC survivor. This second strategy included slides and silicon breast models. These two strategies were then compared to a third one, consisting of a primary care nurse handing out a leaflet and showing a video, based on Health Secretariat's guidelines. In total, 149 women were trained. They were randomly assigned to any one of these three strategies. The effectiveness of the teaching strategies was determined by assessing the changes in the women's knowledge of BC and BSE, as well as in their ability to identify the largest number of lumps on a natural size silicon breast, before and after training. Statistical analysis included Student's test, variance analysis, $M c N$ emar's $\chi^{2}$, Pearson's $\chi^{2}$ and multiple linear regression. ResultsW ith all three strategies, there was an increase of approximately $30 \%$ in women's knowledge of BC and BSE as well as in their ability to detect lumps. Although at
\end{abstract}

* Trabajo financiado por el Consejo N acional de Ciencia y Tecnología, referencias 1371P-M9506 y 111891, por el Programa Especial de Investigación, Desarrollo y Capacitación en Investigación en Reproducción Humana de la O rganización Mundial de la Salud, referencia M8/181/4/0.161; y por The John and C atherine T. Mac Arthur Foundation.

(1) Centro de Investigación en Sistemas de Salud, Instituto N acional de Salud Pública (IN SP), Cuernavaca, Morelos, México.

(2) Centro de Investigación en Salud Poblacional, IN SP, Cuernavaca, Morelos, México.

Fecha de recibido: 15 de junio de 1999 • Fecha de aprobado: 21 de octubre de 1999

Solicitud de sobretiros: M. en C. D oris O rtega Altamirano. Centro de Investigación en Sistemas de Salud, Instituto N acional de Salud Pública. Avenida Universidad 655, colonia Santa María A huacatitlán, 62508 Cuernavaca, Morelos, México.

Correo electrónico: dortega@ insp3.insp.mx 
CM,AES y su destreza para identificar abultamientos. Sólo una de cada 20 mujeres fue capaz de identificar más de tres abultamientos en el modelo al inicio de su capacitación, posteriormente entre 3 y 4 de cada 10 mujeres, valores $p<0.05$. Se identificaron algunos determinantes del aprendizaje del $A E S$, del CM y de la destreza para identificar abultamientos en esta población. Conclusiones La enseñanza del AES con cualquiera de las estrategias evaluadas podría incluirse en un plan integral de prevención y detección temprana del cáncer mamario para las mujeres mexicanas. La práctica adecuada y mensual del AES se vincula tanto con la reducción del lapso entre el hallazgo de un signo sospechoso y la demanda de atención médica, como con la solicitud del examen clínico del seno, lo que implica identificar tumores de menor tamaño cuando se presenta el CM.

Palabras clave: enseñanza; autoexamen del seno; detección oportuna de cáncer; neoplasmas de la mama; México the beginning of the training only one out of every 20 women were able to identify more than three lumps in the silicon model, by the end between 3 and 4 of every 10 women were able to do this, with values of $p<0.05$. Some factors determine women's learning of both BSE and information about BC as well as their ability to identify lumps. Conclusions. Teaching BSE with any of the strategies evaluated could be included in an integral program to educate Mexican women on breast cancer prevention and early detection. The appropriate and monthly practice of BSE is linked both to a decrease in the amount of time elapsing between the identification of suspect signs and the demand for medical care, such as requesting a clinical breast examination, which entails identifying small tumors when $B C$ is present.

Key words: teaching; breast self-examination; early cancer detection; breast neoplasms; Mexico
E 1 cáncer de mama $(\mathrm{CM})$ es un problema de saE lud pública que afecta gravemente a la mujer en edad reproductiva. En México, la mortalidad por esta neoplasia maligna muestra un aumento constante, con un incremento de $74 \%$ en el periodo de $1992^{1}$ a $1997^{*}$ (de 2.9 a 3.9 muertes por 100000 habitantes). Aproximadamente se diagnostican 30 casos nuevos de $\mathrm{CM}$ cada día, ${ }^{2}$ lo que ubica a la enfermedad en el segundo lugar de morbilidad y mortalidad por cáncer en la mujer.

El autoexamen del seno (AES), el examen clínico (EC) y la mamografía son las técnicas disponibles para diagnosticar tempranamente el CM. El tamaño del tumor es un buen indicador de la probabilidad de vivir sin recidivas; la presencia de algún tipo de metástasis (estadios II-III y IV) es proporcional a sus dimensiones. ${ }^{3-7}$ De los casos con metástasis, $47 \%$ presentan tumores de al menos $4.5 \mathrm{~cm}$ de diámetro. ${ }^{6}$

En México, el diagnóstico de CM es tardío. De los casos nuevos $90 \%$ son detectados por la propia mujer en etapas con diseminación ${ }^{1}$ y sólo $2 \%$ se identifican en etapas in situ o estadio I. ${ }^{\ddagger}$

La práctica correcta del AES es un determinante de la demanda oportuna de la atención médica. ${ }^{3}$ En México no se cuenta con información sobre la prevalencia de la utilización del AES o sobre la calidad del mismo.

\footnotetext{
* Dirección General de Epidemiología-Secretaría de Salud (SSA): http://www.ssa.gob.mx/ dgeivital95/vital/cuadro8.html.

‡ López-Carrillo L, Torres-Sánchez L, López-Cervantes M, RuedaNeria C. Diagnóstico temprano del cáncer mamario en México. Enviado a la Revista de Investigación Clínica.
}

Para disponer de una estrategia de enseñanza de la técnica del AES efectiva para las mujeres mexicanas, de entre 12 a 47 años de edad, en el presente estudio se diseñaron y evaluaron dos procedimientos educativos que se compararon con un tercero como referencia. El último fue deducido de la norma y el programa oficial para detectar y controlar el CM.

\section{Material y métodos}

El presente trabajo se realizó de abril de 1996 a julio de 1998, en la ciudad de Cuernavaca, Morelos, México, y constó de cuatro etapas: un estudio cualitativo sobre CM y AES; el diseño de dos estrategias de enseñanza del AES, incluyendo materiales didácticos específicos; la implementación de las estrategias, y la evaluación de las mismas.

\section{Estudio cualitativo}

Se llevaron a cabo cuatro sesiones con la técnica de grupos focales, entre junio y septiembre de 1996, en las que participaron mujeres de entre 12 a 47 años de edad: adolescentes, obreras, madres de familia y maestras. Una antropóloga, especialista en esa técnica, condujo dichas reuniones con duración aproximada de dos horas cada una.

El objetivo de esta fase fue identificar las necesidades de capacitación de las mujeres en CM y en AES; para ello se exploraron los conocimientos, creencias y actitudes en estos aspectos y se evaluaron los contenidos y el formato de materiales didácticos seleccionados que actualmente se utilizan en el Programa Nacional de Prevención y Control del Cáncer Cervi- 
couterino y Mamario de la Secretaría de Salud (SSA). ${ }^{8}$ La metodología y los resultados de esta etapa se describen, en forma extensa, en otro documento.*

\section{Diseño de materiales didácticos y estrategias de enseñanza del AES}

Con base en los resultados del estudio cualitativo descrito previamente, se diseñaron tres nuevos materiales educativos específicos: una secuencia de diapositivas, un folleto y una cinta de video. ${ }^{\ddagger}$ Asimismo, se planearon dos estrategias de enseñanza del AES. Tanto los nuevos materiales como las estrategias diseñadas se describen a continuación.

\section{Secuencia de diapositivas}

En 21 diapositivas a color se incluyeron aspectos relacionados con la magnitud del problema de CM, en términos de su incidencia y mortalidad tanto en México como en Estados Unidos de América (EUA); sus factores de riesgo; las ventajas de las técnicas de detección temprana, y las características del AES, así como las de su práctica.

\section{Folleto}

Se elaboró un folleto informativo, de 16 páginas, con mensajes redactados en lenguaje coloquial sobre qué es el cáncer mamario, los factores que incrementan el riesgo de presentarlo, los hábitos que disminuyen la probabilidad de padecerlo, la anatomía del seno, y los signos diferenciales de esta enfermedad. $\$$ Asimismo, se incluyeron esquemas detallados de la técnica del AES, y las recomendaciones actuales de mayor consenso, sobre la edad y la frecuencia en la que deben practicarse el AES, el EC y la mamografía.

Además, en el folleto se incluyó un cuestionario corto autoaplicable para evaluar el riesgo individual de padecer CM. Este fue una traducción del instrumento elaborado por Spence ${ }^{9}$ que se adaptó de acuerdo con la magnitud de los determinantes reproductivos del CM identificados en mujeres mexicanas. ${ }^{10}$

\footnotetext{
* Ortega-Altamirano D, López-Carrillo L. Bases para una estrategia de capacitación en autoexamen del seno y cáncer mamario en mujeres mexicanas. Documento interno de trabajo.

‡ Para obtener una copia de los materiales diseñados favor de comunicarse con los autores.

\& Ortega-Altamirano D, López-Carrillo L, Tello-Garrido JM. De amiga a amiga. Lo que nosotras necesitamos saber del autoexamen del seno. Instituto Nacional de Salud Pública, México, D.F.: Secretaría de Salud. Documento interno de trabajo.
}

\section{Cinta de video}

La cinta de video titulada Guía para el autoexamen del seno se dobló al español,* en él se presenta de manera visual la información contenida en el folleto descrito en el párrafo anterior.

\section{Estrategia de enseñanza modificada}

En la enseñanza modificada (EM) se consideraron tres componentes: el contenido didáctico, los materiales y la docencia. El primero, incluyó la información básica sobre los determinantes reproductivos, dietéticos y el ejercicio, así como las técnicas de detección temprana de CM. Se utilizaron la cinta de video y el folleto arriba descritos, y una licenciada en enfermería, con experiencia en salud pública, fue la docente, capacitada en AES, por un oncólogo de la mama, y en las técnicas de enseñanza, por una pedagoga especialista en atención primaria a la salud.

\section{Estrategia de enseñanza alternativa}

Además de los componentes considerados en la EM, en la estrategia de enseñanza alternativa (EA) se añadió la enseñanza práctica de la identificación de abultamientos en un modelo de glándula mamaria de silicón con dos nódulos palpables ${ }^{\ddagger}$ y se utilizó, también, la secuencia de diapositivas ya descrita; la docente fue una sobreviviente de CM, y fue capacitada por el mismo personal que la enfermera.

\section{Estrategia de enseñanza tradicional}

La estrategia de enseñanza tradicional (ET) se basó en el Programa Nacional de Prevención y Control del Cáncer Cervicouterino y Mamario de la SSA, ${ }^{8}$ la cual establece que una enfermera capacite a las mujeres en la técnica del AES. Esta capacitación se refiere a la entrega de una hoja impresa, ${ }^{11}$ la presentación de un vi$\mathrm{deo}^{\S}$ y la resolución a las preguntas de las mujeres por parte de la enfermera, especialista en salud pública.

* Ontario Cancer Institute, Princess Margaret Hospital, Bristol-Myers Squibb. Breast self examination guide. A touch of prevention. Cinta de video VHS, color. Duración 8 minutos. Ontario: Debbie Travis, Canadá, 1998.

$\ddagger$ WRS Group. Mini-breast model code 26900. Color beige, $5 \times 7 \mathrm{~cm}$. Waco (TX): Health-Edco, 1996. http://www.wrsgroup.com/ corppgs.

\$ Secretaría de Salud del Estado de Puebla-Grupo Recuperación Total-Reto. Cáncer de mama. Videos Educativos (cinta de video VHS, color. Duración 18 minutos). México, D.F.: SSA, Gobierno del estado de Puebla, 1994. 
En el cuadro I se muestran las características de las dos estrategias propuestas y la de referencia.

\section{Implementación de las estrategias}

Durante julio de 1998 se llevó a cabo un ensayo comunitario con las dos estrategias de enseñanza del AES propuestas (EM y EA) y la de referencia (ET), se asignó aleatoriamente a cada una de las participantes a una de las tres estrategias.

Participaron 149 mujeres en total: 23 adolescentes, de entre 14 a 21 años, estudiantes de una escuela técnica; 47 trabajadoras, de entre 18 a 48 años de edad,

\section{Cuadro I \\ Características de las tres estrategias de enseñanza del autoexamen del seno para mujeres en edad reproductiva. Cuernavaca, Morelos, Méxıco, 1998}

Características/estrategias

Referencia tradicional Modificada Alternativa

Contenido didáctico

\begin{tabular}{llll} 
Etiología & $\mathrm{x}$ & $\mathrm{x}$ & $\mathrm{x}$ \\
\hline Prevención & & $\mathrm{x}$ & $\mathrm{x}$ \\
\hline Diagnóstico & $\mathrm{x}$ & $\mathrm{x}$ & $\mathrm{x}$
\end{tabular}

Materiales

Hoja impresa (1 hoja)

\begin{tabular}{|c|c|c|c|}
\hline Folleto (16 hojas) & & $x$ & $\mathrm{x}$ \\
\hline Cinta de video 8 min* & & $\mathrm{x}$ & $\mathrm{x}$ \\
\hline Cinta de video $18 \mathrm{~min}^{\ddagger 5}$ & $x$ & & \\
\hline Secuencia de diapositivas & & & $\mathrm{x}$ \\
\hline
\end{tabular}

Docente

\begin{tabular}{|c|c|c|c|}
\hline Enfermera & $x$ & $x$ & \\
\hline Sobreviviente & & & $x$ \\
\hline
\end{tabular}

Sesiones de capacitación

\begin{tabular}{llll} 
Duración: 1 hora 15 minutos & $\mathrm{x}$ & $\mathrm{x}$ & $\mathrm{x}$ \\
\hline N úmero: cuatro grupos & $\mathrm{x}$ & $\mathrm{x}$ & $\mathrm{x}$
\end{tabular}

Técnicas didácticas utilizadas

Exposición $\quad x \quad x \quad x$

Presentación de cinta de video Interrogatorio de las participantes a las docentes

* Presenta mujeres con tres tipos de senos realizándose el AES: una de mediana edad y obesa; otra joven con senos pequeños y firmes, y una añosa con senos en forma de péndulo

* Presenta una mujer con senos firmes y pequeños realizándose el AES

$\S$ Incluye tratamientos: cirugía, radioterapia y quimioterapia

\# Adolescentes, obreras, maestras y madres de familia obreras de una fábrica de productos electrónicos; 44 madres de familia de una colonia popular selecciona$\mathrm{da}$, de entre 21 a 48 años de edad, y un grupo de 35 maestras, de un centro de investigación científica, de entre 22 a 46 años. Todas fueron captadas en la ciudad de Cuernavaca.

Las instituciones (escuela, fábrica y centro de investigación) y la colonia popular fueron seleccionadas por contar con población femenina de entre 12 a 47 años, y por disponer de instalaciones adecuadas para realizar la capacitación.

Además de la edad, los criterios de inclusión fueron: no tener algún cáncer diagnosticado a la fecha del estudio y haber residido por lo menos durante un año previo en la ciudad donde se realizó el estudio.

Se obtuvo la información sobre los aspectos para evaluar las estrategias de enseñanza del AES por medio de un cuestionario aplicado a todas las participantes, previamente a su asignación aleatoria. $\mathrm{Al}$ salir de la única sesión de entrenamiento se les aplicó nuevamente dicho cuestionario.

\section{Evaluación de las estrategias de enseñanza del AES}

Para determinar la eficacia de cada estrategia se calificaron tres aspectos: los conocimientos generales sobre la etiología, prevención y diagnóstico temprano del $\mathrm{CM}$, los conocimientos de la técnica del AES y la destreza para identificar abultamientos en un modelo de glándula mamaria de silicón de tamaño natural. ${ }^{*}$ Adicionalmente, se obtuvo información sobre las actitudes y creencias de las participantes respecto de $\mathrm{CM}$ y el AES, así como para la evaluación de los componentes didácticos de las estrategias.

Se otorgó un máximo de 23 puntos en los conocimientos generales del $\mathrm{CM}$ que correspondió a las respuestas correctas del mismo número de preguntas. Los conocimientos de la técnica del AES (la revisión visual, la manual de pie y acostada $)^{12-15}$ fueron explorados en 13 preguntas y se otorgó un punto por cada una que estuviera correcta. La destreza para identificar abultamientos se calificó con cinco puntos como máximo si se encontraban los cinco contenidos en el modelo de glándula mamaria de silicón.*

Se incluyeron 12 afirmaciones relacionadas con actitudes ante la prevención de CM y la práctica del

\footnotetext{
* WRS Group Inc. Breast-self examination model code GS26513. Color beige, 29x17 cm. Waco (TX): Health-Edco, 1996. http:// www.wrsgroup.com/corppgs.
} 
AES y 12 más relacionadas con las creencias sobre la etiología y el pronóstico de esta enfermedad. De estas últimas, 10 se basan en las creencias identificadas en las mujeres durante el estudio cualitativo antes descrito y aluden a factores inadmisibles. Se crearon dos índices, uno de calificación total de actitudes y creencias con valor máximo de 24 puntos y el otro, de 10, con sólo creencias.

Cada estrategia fue evaluada por las participantes en términos del desempeño docente y de las actividades de enseñanza (40 puntos); el contenido temático (60 puntos); los recursos didácticos empleados (180 puntos), y la apreciación del propio aprendizaje y de las actividades efectuadas durante la capacitación (30 puntos). La calificación total máxima posible fue de 310 puntos.

\section{A nálisis estadístico}

El análisis estadístico incluyó la comparación de las medias de calificaciones antes y después de que se implementaran cada una de las estrategias, mediante la prueba $t$ de Student, del análisis de varianza y de las pruebas $\chi^{2}$ de McNemar y la de Pearson. Ade- más, se identificaron los determinantes de los conocimientos sobre el CM y el AES, y de la destreza para identificar abultamientos por medio de regresión lineal múltiple. El análisis fue efectuado con el paquete estadístico Stata 5.0 (Stata Corporation, College Station, TX).

\section{Resultados}

En el cuadro II se muestran las características generales de todas las participantes. En promedio tuvieron, aproximadamente, 29 años de edad, 12 años de escolaridad y 19 años de vivir en la zona del estudio, cifras que resultaron similares en cada subgrupo después de la asignación aleatoria. Asimismo, cerca de $72 \%$ de la población de estudio, informó tener una ocupación remunerada y $82 \%$, tener derecho a servicio médico, lo que no varió en los grupos de intervención. Se obtuvo un porcentaje mayor de mujeres solteras en el grupo de la ET, lo que fue estadísticamente significativo a través de los grupos.

La evaluación de las estrategias de enseñanza se presenta en el cuadro III. Las calificaciones obtenidas por todas las mujeres inicialmente, en los grupos

\section{Cuadro II \\ Características sociodemográficas de la población de estudio, por estrategia de enseñanza y total. Cuernavaca, Morelos, México, 1998}

\begin{tabular}{|c|c|c|c|c|}
\hline \multirow[t]{2}{*}{ Característica } & \multicolumn{3}{|c|}{ Estrategia de enseñanza } & \multirow[b]{2}{*}{ Población total $(n=149)$} \\
\hline & Tradicional $(n=52)$ & Modificada $(n=45)$ & Alternativa $(n=52)$ & \\
\hline \multicolumn{5}{|l|}{ Edad (años) } \\
\hline Media & 28.94 & 28.20 & 29.96 & 29.07 \\
\hline Mín-máx & $16-48$ & $14-48$ & $16-48$ & 14-48 \\
\hline \multicolumn{5}{|c|}{ Escolaridad (años) } \\
\hline Media & 12.13 & 11.68 & 13.17 & 12.36 \\
\hline Mín-máx & $1-25$ & $3-21$ & $9-25$ & $1-25$ \\
\hline \multicolumn{5}{|c|}{ Tiempo de vivir en Cuernavaca (años) } \\
\hline Media & 21.11 & 18.48 & 16.73 & 18.79 \\
\hline Mín-máx & 4-41 & $1-46$ & $2-45$ & $1-46$ \\
\hline \multicolumn{5}{|l|}{ Estado civil* } \\
\hline Soltera \% & 63.46 & 37.78 & 40.38 & 47.65 \\
\hline Casada \% & 36.54 & 62.22 & 59.62 & 52.35 \\
\hline \multicolumn{5}{|c|}{0 cupación remunerada } \\
\hline Sí (\%) & 73.08 & 68.89 & 75.00 & 72.48 \\
\hline \multicolumn{5}{|c|}{ Derecho a servicio médico } \\
\hline Sí (\%) & 82.69 & 82.22 & 82.69 & 82.55 \\
\hline
\end{tabular}




\section{Cuadro III \\ Conocimientos de las mujeres en CÁnCer mamario, AUtoexamen del SENo Y destreza para IDENTIFICAR ABULTAMIENTOS, SEGÚN LA ESTRATEGIA DE ENSEÑANZA Y EVALUACIÓN DE LA CAPACITACIÓN. Cuernavaca, Morelos, México, 1998}

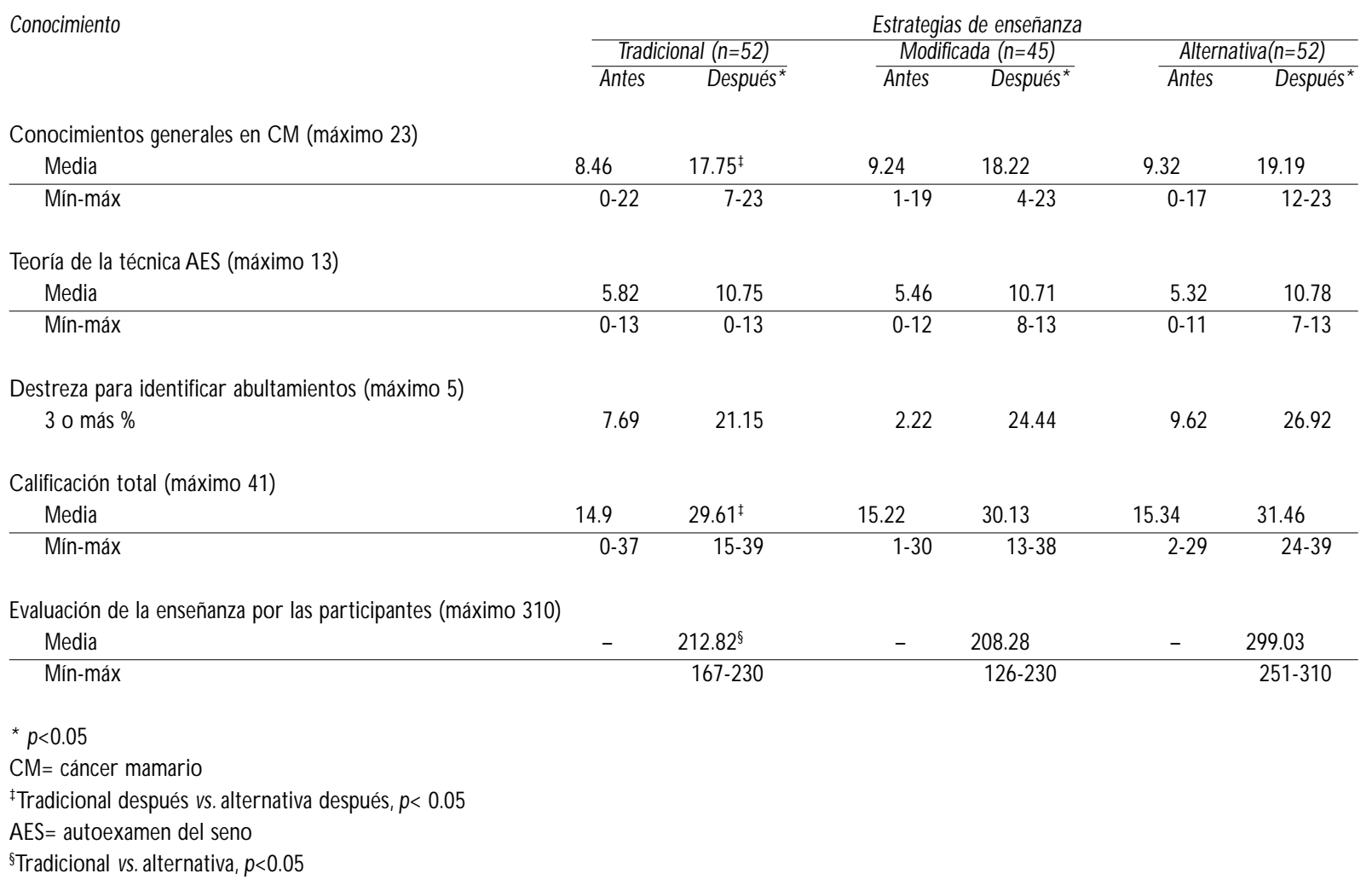

de intervención, fueron muy similares en cada uno de los aspectos evaluados. Después de la capacitación los puntajes respectivos fueron significativamente mayores en los tres grupos. El cambio proporcional observado en la calificación total antes y después de llevar a cabo la ET fue 35.87\%, mientras que en la EM, de $36.36 \%$ y en la EA, de $39.31 \%$.

Los factores que determinaron proporcionalmente y de manera significativa el aprendizaje de los conocimientos evaluados fueron la edad, la escolaridad, el presentar más actitudes positivas y menos creencias en causas inadmisibles de CM, así como tener una actividad remunerada. La destreza para identificar abultamientos aumentó significativamente al incrementarse el tiempo empleado en la revisión del modelo de glándula mamaria de silicón ${ }^{19}$ y el riesgo de presentar la enfermedad. Los modelos estimados explicaron de 18 a $37 \%$ de la varianza (cuadro IV).

En cuanto a las creencias en factores de riesgo inadmisibles de CM, en la figura 1 se muestra la dis- tribución proporcional antes y después de cada intervención. Nuevamente, la reducción mayor y significativa se obtuvo en el grupo de la EA (4.25 antes a 0.63 puntos después).

\section{Discusión}

La enseñanza con cualquiera de las técnicas evaluadas, capacitó aproximadamente a 30\% de las participantes para identificar abultamientos y elevó sus conocimientos en CM y AES. Los principales hallazgos de este estudio muestran que una de cada $20 \mathrm{mu}-$ jeres es capaz de identificar más de tres abultamientos de 0.5 a $1 \mathrm{~cm}$ en el seno y que esta destreza se incrementó significativamente de 3 a 4 de cada 10 mujeres con las estrategias de enseñanza de la técnica del AES.

La proporción de mujeres capaces de identificar al menos un abultamiento varía de 25 a $88 \%$ en otros estudios similares llevados a cabo principalmente en EUA. En el presente estudio, la proporción corres- 


\section{Cuadro IV \\ Determinantes del aprendizaje de las mujeres en Cáncer mamario, en autoexamen del Seno y en destreza para identificar abultamientos en un modelo de silicón.* Cuernavaca, Morelos, México, 1998}

\begin{tabular}{|c|c|c|c|c|}
\hline Aprendizaje & Determinante & Coeficiente & p & $r^{2}$ \\
\hline \multirow[t]{2}{*}{ Conocimientos generales en $\mathrm{CM}$} & Escolaridad & 0.346 & 0.000 & 0.264 \\
\hline & Tener actitudes positivas y menos creencias inadmisibles & 4.070 & 0.082 & \\
\hline \multirow[t]{3}{*}{ Teoría de la técnica AES } & Edad & 0.041 & 0.020 & \\
\hline & Realizar una actividad remunerada & 0.943 & 0.004 & 0.271 \\
\hline & Tener actitudes positivas y menos creencias inadmisibles & 0.152 & 0.000 & \\
\hline \multirow[t]{2}{*}{ Destreza para identificar abultamientos } & Tiempo empleado en la revisión del MGMS & 0.635 & 0.000 & 0.182 \\
\hline & Riesgo de presentar la enfermedad & 0.073 & 0.005 & \\
\hline \multirow[t]{3}{*}{ Calificación total } & Escolaridad & 0.344 & 0.000 & 0.376 \\
\hline & Realizar una actividad remunerada & 1.812 & 0.021 & \\
\hline & Tener actitudes positivas y menos creencias inadmisibles & 0.382 & 0.000 & \\
\hline
\end{tabular}

* Ajustado por estrategia de enseñanza y estado civil

$\mathrm{CM}=$ cáncer mamario

$A E S=$ autoexamen del seno

MGMS= modelo de glándula mamaria de silicón

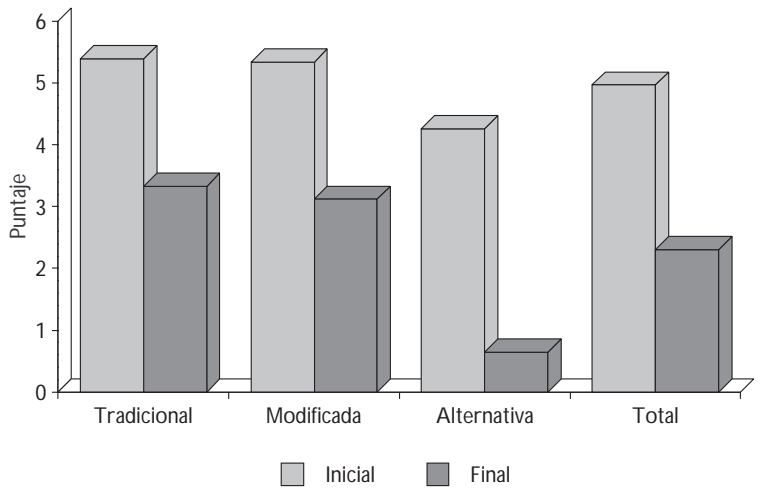

Figura 1. Creencias inadmisibles de las mujeres en FACTORES DE RIESGO DEL CÁNCER MAMARIO, POR EStrategia de enseñanza y total. Cuernavaca, MoreLOS, MÉXICO, 1998

pondiente después del entrenamiento fue de 51 a $65 \%$ de acuerdo con el grupo de intervención, las cuales son similares a las ya notificadas en países desarrollados. ${ }^{3,4,16-19}$

En algunos países existen acciones educativas en AES que han mostrado buenos resultados.,13,14,20,21 $\mathrm{La}$ mayoría se basan en los servicios de atención de primer nivel, donde el personal de enfermería ofrece el entrenamiento a las mujeres que asisten a dichos servicios. ${ }^{13,21}$ Otras estrategias efectivas incluyen tener personal técnico específicamente adiestrado para visitar a las mujeres en sus domicilios y enseñarles la técnica. ${ }^{4,20}$ Cada una de las modalidades está en función de los recursos, la magnitud del problema y las políticas de salud vigentes en cada país.

Se ha comprobado que las campañas a través de los medios masivos de comunicación y la distribución de material impreso aumentan los conocimientos generales de CM; sin embargo, están lejos de propiciar un cambio efectivo en el número de mujeres que se realizan el AES o en el de abultamientos que éstas pueden identificar; sólo incrementan los costos de los servicios médicos. ${ }^{4,22,23}$

Actualmente se encuentra en revisión la Norma Oficial Mexicana NOM-014 SSA2-1994, en la que se establece que el AES debe enseñarse en las unidades de primer nivel de atención a la salud por el personal de enfermería. ${ }^{8,24} \mathrm{Al}$ respecto, no se cuenta con información sobre la frecuencia con la que se ha ofrecido la capacitación a las usuarias y menos aún de la calidad de la enseñanza de la técnica en este nivel.

Con base en los resultados de este estudio, se puede sugerir que la EA sea evaluada en México, a nivel poblacional, en términos de su costo y efectividad; para ello se propone involucrar en forma voluntaria a las sobrevivientes de CM para enseñar la 
técnica; ellas podrían ser reclutadas en los mismos servicios de salud o a través de otros grupos y organizaciones ya existentes.*

Adicionalmente, los materiales didácticos diseñados específicamente podrían utilizarse, previo entrenamiento, por el personal técnico adscrito al programa vigente de prevención y control del cáncer cervicouterino. Es decir, se optimizarían los recursos invertidos en ese programa de tal forma que el personal podría realizar una doble función, tomar la muestra para el Papanicolaou y enseñar el AES, con un costo marginal mínimo.

La enseñanza de la prevención de cáncer alrededor del inicio de la adolescencia efectuada en este estudio es una estrategia recientemente utilizada en algunos países como Uruguay. ${ }^{25}$ En el caso de CM se ha argumentado que el aprendizaje temprano del AES es una forma efectiva de sensibilizar y desarrollar el conocimiento de los propios senos. ${ }^{18,26}$ En México, se ha incluido un capítulo sobre prevención de cáncer cervicouterino y mamario en El manual para el maestro, el que se utilizará a partir del próximo ciclo escolar en la enseñanza media. ${ }^{\ddagger} \mathrm{El}$ impacto de esta innovación eventualmente se tendrá que evaluar.

Si bien es importante que las mujeres aprendan la técnica del AES, también lo es que la practiquen mensualmente. ${ }^{3,4,20,26}$ Es decir, es necesario evaluar la adecuación con que se efectúe dicha práctica, aspecto que no se estudió en el presente trabajo.

El impacto de la utilización del AES en las tasas de mortalidad de CM en el ámbito mundial ha mostrado resultados inconsistentes. En un estudio longitudinal realizado en Finlandia en $1993^{27}$ se estimó que el AES reduce la mortalidad al identificar tumores en etapas I/II previas a la presencia de metástasis; no obstante, la mayoría de las investigaciones no han informado una disminución significativa de la mortalidad por CM atribuible a la utilización de la técnica, pero sí a factores vinculados a ésta, como es el acortamiento del lapso entre el que la mujer encuentra un abultamiento en sus senos y acude a solicitar asistencia médica, ${ }_{17}^{17} \mathrm{o}$ el de demandar anualmente el EC. ${ }^{\$, 28}$ Ambos

\footnotetext{
* Grupo Recuperación Total (RETO), organización constituida por mujeres sobrevivientes de cáncer mamario. Benjamín Franklin 61-D, colonia Condesa, México, D.F. Tel.: 52-77-78-74 y 5272-70-08.

‡ López-Carrillo L, Torres-Sánchez L. Prevención del cáncer cérvico-uterino y mamario. En: Langer A, comp. Prevención del cáncer. Manual para el Maestro. México, D.F.: EDAMEX, 1999.

§ López-Carrillo L, Torres-Sánchez L, López-Cervantes M, RuedaNeria C. Diagnóstico temprano del cáncer mamario en México. Enviado a la Revista de Investigación Clínica.
}

aspectos se vinculan con un mejor pronóstico en caso de presentar el padecimiento.

Los estudios sobre el impacto del AES en la mortalidad por CM presentan limitaciones metodológicas importantes como: el sesgo de memoria (recall bias) al obtener información sobre la práctica del AES en mujeres que han presentado la enfermedad; inexactitud en los informes de las mujeres respecto de la práctica de la técnica (adecuación y oportunidad); así como dificultades para la medición de su efectividad. ${ }^{4,22}$ Por lo anterior, es necesario contar con los resultados del estudio multicéntrico de la Organización Mundial de la Salud que actualmente se está llevando a cabo en forma longitudinal. ${ }^{29}$

El AES no resuelve completamente la necesidad de realizar un diagnóstico más temprano de los casos de $\mathrm{CM}$; sin embargo, puede incluirse en una estrategia integral de prevención y tamizaje, por ejemplo al conjuntarla con la práctica del EC anual, hasta que sea factible contar con técnicas de detección temprana más efectivas y accesibles para todas las mujeres.

\section{Agradecimientos}

Los autores agradecen las aportaciones técnicas de José Manuel Tello-Garrido; las facilidades para la realización del trabajo a Jaime Sánchez, Rocío Romero, Donita y Marie Porter, Martha Rivera, Shirley Velasco, Leticia Martínez, Martha Sánchez, Patricia Veloz, Rocío Osorio, Patricia Mainou, María Esther Rivera y Elida Velasco, y la donación de 10 minimodelos a la Health Edco, Waco, Tejas, EUA.

\section{Referencias}

1. 0 laiz G, Hernández M. Cáncer, un encuentro con el futuro epidemiológico. En: Sepúlveda J, coord. Los retos de la transición. Cuadernos de Salud núm. 1. México, D.F.: SSA, 1994:81.

2. Dirección General de Epidemiología, Secretaría de Salud. Registro histopatológico de neoplasias en México, 1993-1996. México, D.F.: SSA, 1997:25.

3. Senie R, Rosen PP. Breast self examination and medical examination related to breast cancer stage. Am J Public Health 1981;71:583-590.

4. Pinto B, Fuqua W R. Training breast self examination: A research review and critique. Health Educ Q 1991;18:495-516.

5.A merican Joint C ommittee on C ancer. Handbook for staging of cancer, from the manual for staging of cancer. Filadelfia: Lippincott, 1993:280.

6. Gloeckler-Ries LA, Earl-Henson D, H arras A. Survival from breast cancer according to tumor size and nodal status. Medicolegal controversies in breast cancer : Biologic basis and risk prevention. Surg $\mathrm{O}$ ncol $\mathrm{C}$ lin N orth Am 1994;3(I):35-65.

7. 0 steen $R, W$ inchester $D, C$ unningham $M$. Breast cancer. En: Steele $G$, Jessup $M, W$ inchester $D, M$ enck $H$, Murphy $G$, comp. N ational cancer data- 
base/Annual review of patient care breast cancer.Atlanta (GA):American Cancer Society, 1995;12-35.

8. Dirección General de Atención Materno Infantil, Dirección General de Medicina Preventiva. Programa Nacional de Prevención y Control del Cáncer Cervicouterino y Mamario. México, D.F.: Secretaría de Salud, 1992:52.

9. Spence W R. Good N ews. W aco (TX): Health-Edco, 1996:16.

10. López-Carrillo L, Bravo-Alvarado J, Poblano-Verástegui 0, 0 rtega-Altamirano D. Reproductive determinants of breast cancer in Mexican women. Preventive strategies for living in a chemical world. Ann N Y Acad Sci 1997;837:537-550.

11. Dirección General de Salud Materno-Infantil, Secretaría de Salud/Fondo de las $\mathrm{N}$ aciones Unidas para la Infancia/O rganización Mundial de la Salud, 0 rganización Panamericana de la Salud. La salud empieza en casa. Cáncer mamario. México, D.F.: SSA, 1995:1.

12. Pennypacker HS, Goldstein MK, Stein GH. A precise method of manual breast self examination. En: Mettlin C, Murphy GP, comp. Progress in Cancer Control IV. Research in The Cancer Center. N ueva York:Alan R Liss, 1983:305-311.

13. Assaf A, C ummings M, G raham S, Mettlin C, Marshall J. Comparison of three methods of teaching women how to perform breast self examination. Health Educ Q 1985;12:259-272.

14. Carter A, Feldman J,T iefer L, Hausdorff J. Methods of motivating the practice of breast self examination: A randomized trial. Prev Med 1985; 14:555-572.

15. Celentano D, Holtzman D. Breast self examination competency: An analysis of self reported practice and associated characteristics. Am J Public Health 1983;73:1321-1323.

16. Pennypacker HS, Bloom HS, Criswell E, N eelakantan P, Goldstein M, Stein $G$.Toward an effective technology of instruction in breast self examination. Int J Mental Health 1982;11:98-116.

17. Huguley $\mathrm{Ch}$, Brown R. The value of breast self examination. Cancer 1981;47: 989-995.
18. Love S, Lindsey K. Getting acquainted with your breast. En: Love S, Susan Love's breast book. 2a ed. Indianapolis:Addison-W esley Publishing Company, 1995:19-25.

19. G reenwald P, N asca P, Lawrence C, H orton J, McG arrah R, Gabriele T et al. Estimated effect of BSE and routine physician examination on breast cancer mortality. N Engl J Med 1978;299:271-273.

20. Fletcher S, 0 'Malley M, Earp J, Morgan T, Lin S, D egnan D. How best to teach women breast self-examination.Ann Intern Med 1990;112:772-779. 21. Worden J, Solomon L, Flynn B. A community wide program in breast self examination trainning and maintenance. Prev Med 1990;19:254-269. 22. Morrison AS. Screening for cancer of the breast. Epidemiol Rev 1993; 15:244-255.

23. C alnan M, Moss S.The health belief model and compliance with education given at a class in breast self examination.J Health Soc Behav 1984; 25:198-210.

24. Diario 0 ficial. N orma 0 ficial Mexicana N O M-014-SSA2-1994 para la prevención, tratamiento y control del cáncer del cuello del útero y de la mama en la atención primaria. Secretaría de Salud-SSA. Lunes 16 de enero de 1995.2ª sec.:1-11.

25. Ministerio de Salud. Medidas para prevenir el cáncer. G uía para niños y jóvenes. Montevideo: Ministerio de Salud, 1997:1-16.

26. Hill D,W hiteV, Jolley D, H apperson K. Self examination of the breast: Is it beneficial? Meta-analysis of studies investigating breast self examination and extend of disease in patiens with breast cancer. $\mathrm{Br} \mathrm{M} \mathrm{J}$ 1988;297: 271-275.

27. Miller $A B$.W hat is the role of early detection and screening in cancer control? J Public Health Policy 1993:403-411.

28. Foster RS. Limitations of physical examination in the early diagnosis of breast cancer. Surgycal 0 ncology C linics of N orth America. 1994;1:55-65. 29. Semiglazov VF, Moiseenko VM. Breast self examination for the early detection of breast cancer: AUSSR/W HO controled trial in Lenningrad. Bull W orld Health O rgan 1987;65:391-396. 